\title{
Modulating myelination: knowing when to say Wnt
}

\author{
Sheila S. Rosenberg and Jonah R. Chan ${ }^{1}$ \\ Department of Biochemistry and Molecular Biology, Zilkha Neurogenetic Institute, Keck School of Medicine at the University \\ of Southern California, Los Angeles, California 90033, USA
}

\begin{abstract}
Balancing extrinsic and intrinsic cues plays an integral part in shaping the complex architecture of the nervous system. During development, oligodendrocyte precursor cells integrate environmental signals and coordinate the activation of the transcriptional machinery necessary for differentiation and myelination. In this issue of Genes \& Development, Fancy and colleagues (pp. 1571-1585) demonstrate that canonical Wnt signaling contributes to the decision of an oligodendrocyte precursor cell to differentiate-both during development and after demyelination. These findings provide new insight into the precise spatiotemporal coordination required for oligodendrocyte development.
\end{abstract}

Balancing proliferation and differentiation: a numbers game

The likely success or failure of most tasks can be summed up in one key phrase: "Timing is everything." This same principle can be applied to developmental cell biology. Precisely orchestrated cues ensure a carefully coordinated balance between fundamental processes such as proliferation, migration, differentiation, and apoptosis. The regulation of these processes requires extreme temporal specificity to ensure both the necessary diversity and the appropriate numbers of each cell type required for a functional output. In the CNS, the development of myelinating oligodendrocytes represents a classic example of the importance of rigorously timed transitions.

Myelination by oligodendrocytes ensures the rapid and efficient propagation of action potentials throughout the CNS. Generating the appropriate number of oligodendrocytes is therefore essential for proper communication between neurons. Oligodendrocytes represent a terminally differentiated, nonproliferative population of cells. For this reason the number of differentiated oligodendrocytes is highly dependent on the extent of proliferation that occurs at the level of the oligodendrocyte precursor cell (OPC). During development, OPCs are exposed to

[Keywords: Oligodendrocyte; CNS development; Wnt; multiple sclerosis; remyelination; Olig2]

${ }^{1}$ Corresponding author.

E-MAIL: jonah.chan@usc.edu; FAX: (323) 442-2448.

Article is online at http://www.genesdev.org/cgi/doi/10.1101/gad.1824009. proliferative signals as they migrate along axons throughout the CNS. Eventually these precursors reach a final destination, and the vast majority will differentiate into myelinating oligodendrocytes. The final number of myelinating cells is determined by the extent of OPC proliferation, as well as the number of OPCs that ultimately differentiate. If proliferation terminates prematurely, the number of OPCs available for differentiation will not be sufficient to successfully populate the CNS and deficits may result. On the other hand, delayed differentiation can also lead to hypomyelination and impaired signaling. It is therefore essential that the transition from OPC to oligodendrocyte be temporally coordinated. Identifying the factors that regulate this transition is important not just for understanding development but also for therapeutic treatment of demyelinating diseases, particularly in light of growing evidence suggesting that OPCs are involved in remyelination (Gensert and Goldman 1997; Levison et al. 1999; Nishiyama et al. 1999; Horner et al. 2000; Levine et al. 2001; Chari and Blakemore 2002; Watanabe et al. 2002; Dawson et al. 2003; Windrem et al. 2004; Rivers et al. 2008).

The majority of OPCs generated during development will differentiate into myelinating oligodendrocytes. However, a small but significant proportion of OPCs remain as progenitors into adulthood. Following the death of oligodendrocytes due to injury or disease, these precursors can be mobilized to remyelinate lesioned areas and prevent further deficits in signal propagation. Unfortunately, the capacity for repair is limited and ultimately fails in the case of chronic demyelinating diseases such as multiple sclerosis (MS). Increasing evidence suggests that the ultimate failure of remyelination may be due not to a deficit of available precursors, but to an inhibition of differentiation (Back et al. 2005; Kotter et al. 2006; Mi et al. 2007; Vargas and Barres 2007). The precise reasons for this limitation remain to be elucidated. In this issue of Genes \& Development, an intriguing study by Fancy et al. (2009) provides new insight into this fundamental question.

The differentiation roadblock: contributions from intrinsic and extrinsic factors

Like any complex developmental process, the onset of myelination requires precise coordination between intrinsic 
and extrinsic cues. During development, a careful balance between these two influences results in perfectly myelinated axon tracts. But what happens when things go awry in the case of demyelination following nerve injury or disease? Growing evidence points to the transition from precursor to differentiated cell as the major hurdle preventing successful remyelination. What is responsible for inhibiting differentiation and what is required to overcome this inhibition in order to allow remyelination to proceed? To properly address these questions, two specific hypotheses need to be considered. One possible explanation is that environmental cues are preventing the OPCs from successfully differentiating and remyelinating. Both the presence of inhibitory cues and an absence of inductive cues could result in limited differentiation. Equally important to consider is the possibility that intrinsic changes to the OPCs themselves are responsible for the failure to successfully transition into oligodendrocytes. Most likely, it is a combination of both environmental and intrinsic factors that is responsible for the arrested development of adult OPCs. In their current study, Fancy et al. (2009) employ a novel genome-wide screen to examine how these factors work together to regulate the remyelination process. Following chemical induction of demyelination in the adult mouse spinal cord, Fancy et al. (2009) examine lesioned tissue for changes in expression of 1040 putative transcription factors. A secondary screen, involving colocalization between in situ hybridization and immunohistochemical analysis, identifies the location of transcription factor expression in specific cell types.

The elegant approach employed by Fancy et al. (2009) facilitates a detailed characterization of the demyelinated environment. As emphasized by the results of this study, cells of the oligodendrocyte lineage are not the only cell type affected by the onset of demyelination. On the contrary, the entire milieu of the adult CNS is likely to be altered by the presence of a demyelinating insult. The results of the screen by Fancy et al. (2009) are consistent with this idea, and demonstrate altered expression patterns of transcription factors found in multiple cell types. To rule out any region-specific changes in expression, Fancy et al. (2009) repeat the screen using multiple demyelination models and examine lesioned tissues in various areas of the CNS. The robustness of this screening method provides a unique opportunity to characterize both extrinsic and intrinsic regulators of remyelination by adult OPCs. Future studies examining additional screening candidates will no doubt prove useful for characterizing environmental factors affecting the differentiation process. However, in their study, Fancy et al. (2009) focus primarily on intrinsic changes that may be responsible for inhibiting oligodendrocyte differentiation.

\section{It's what's inside that counts: transcriptional regulation of oligodendrocyte differentiation}

Transitioning from a precursor cell to a differentiated fate requires precise transcriptional timing and coordination. In the case of the oligodendrocyte, the down-regulation of proliferative and migratory genes is seamlessly integrated with the activation of genes required for the elaboration of the myelin sheath. Disruptions to either the efficacy or the timing of either of these transcriptional components could be extremely detrimental to the remyelination process. The screen by Fancy et al. (2009) could therefore prove extremely useful in determining which transcription factors are important and their individual contributions to myelination. Fancy et al. (2009) identify Tcf4, whose expression is up-regulated in OPCs following demyelination, as a potential regulator of the differentiation process. This represents an intriguing possibility because Tcf4 is involved in the transcriptional regulation of Wnt pathway target genes, and Wnt signaling has been implicated recently in embryonic oligodendrocyte development (Shimizu et al. 2005). To test this hypothesis, Fancy et al. (2009) examine the expression pattern of Tcf4 during postnatal oligodendrocyte development. Tcf4 is expressed in the white matter of the mouse spinal cord during the course of developmental myelination, between postnatal day $1(\mathrm{P} 1)$ and P15. Tcf4 expression is subsequently down-regulated, and is absent from the white matter of adult mice. This expression pattern is consistent with the possibility that Tcf4 is involved in regulating oligodendrocyte differentiation.

It is interesting to note that Tcf4 has been implicated in the formation of multiple nucleoprotein regulatory complexes, allowing it to function as both an activator and an inhibitor of transcription. In the canonical Wnt signaling pathway, Tcf4 associates with $\beta$-catenin to form a complex that is typically associated with gene activation (van de Wetering et al. 2002). Initially, Fancy et al. (2009) verify the presence of $\beta$-catenin/Tcf4-mediated Wnt signaling in the oligodendrocyte lineage. Fancy et al. (2009) confirm that canonical Wnt signaling is active in OPCs-both during development and in lesioned adult tissue. To address whether this signaling pathway plays a role in oligodendrocyte differentiation, Fancy et al. (2009) create transgenic mice characterized by dominant-active (DA) $\beta$-catenin signaling. In these mice, Olig2-driven Cre recombinase is responsible for the expression of a degradationresistant version of $\beta$-catenin. Using this approach, it is possible to induce stable, continuous expression of $\beta$-catenin in oligodendrocyte lineage cells. Fancy et al. (2009) use these mice to test whether dysregulation of $\beta$-catenin/Tcf4-mediated Wnt signaling affects developmental oligodendrocyte differentiation and myelination. Interestingly, beginning at P6 and continuing until P20, these transgenic animals exhibit a strong phenotype characterized by tremor and ataxia, phenotypic traits suggestive of dysmyelination. Indeed, immunohistochemical staining and electron microscopy (EM) studies display a significant reduction in the number of myelinated axons in the developing mouse spinal cord of the DA- $\beta$-catenin mice. Additionally, the axons that were myelinated had significantly fewer wraps of myelin as compared with the axons in control mice. However, this initial deficit in myelination is resolved by $\mathrm{P} 60$, at which point the extent of myelination is equivalent to that of control animals. These results suggest that while DA- $\beta$-catenin signaling 
results in severe hypomyelination, this developmental delay ultimately can be overcome. Fancy et al. (2009) suggest that this functional improvement is due to the eventual down-regulation of Tcf4, which normally occurs around $\mathrm{P} 30$. In the absence of its obligate binding partner, DA- $\beta$-catenin is no longer capable of regulating transcription. Based on the results presented here, as well as recent findings implicating Tcf4 as a negative regulator of myelin gene expression (He et al. 2007), Fancy et al. (2009) postulate that the down-regulation of Tcf4 allows for the expression of myelin genes and subsequent differentiation. While this hypothesis is reasonable, the role of Tcf4 as a dual mediator of transcription invites further analysis of the effect of this transcription factor on differentiation.

If the $\beta$-catenin/Tcf4 complex traditionally functions to activate genes, then how does Tcf 4 negatively regulate myelin gene expression? If Tcf4 serves to activate factors involved in the inhibition of myelin gene expression, this could suggest a potential mechanism by which Tcf $4 / \beta$ catenin-mediated transcription can negatively regulate differentiation (He et al. 2007). However, the putative function of Tcf4 as an active repressor of transcription should not be overlooked. In fact, Fancy et al. (2009) show that OPCs also exhibit colocalization between Tcf4 and Groucho, a member of the Tle family of proteins. The complex formed between these two proteins can repress Wnt target genes. This suggests that Tcf4 could be regulating differentiation through transcriptional repression, although it is also possible that the association between Tcf4 and Groucho is involved in the regulation of other aspects of OPC development. It will be interesting to determine the precise role of this alternate Tcf4 complex in oligodendrocyte development. Fancy et al. (2009) demonstrate that Tcf4 is expressed throughout the course of developmental myelination. If Tcf 4 is involved solely in the inhibition of differentiation, how can it be expressed continuously, albeit only in a subpopulation of cells, when myelination is actively occurring? This potential paradox illustrates the importance of further studies characterizing the role of Tcf4 in the myelination process.

\section{Wnt signaling and demyelination}

The intriguing results of these mouse studies conducted by Fancy et al. (2009) suggest that $\beta$-catenin/Tcf4-mediated Wnt signaling plays an essential role in the proper timing of oligodendrocyte differentiation. While these results are significant in their own right, the importance of these findings is elevated by the fact that Fancy et al. (2009) are able to show that the temporal expression pattern of Tcf4 is conserved in human tissue, both during development and in lesioned tissue from MS patients. In humans, the pattern of Tcf4 expression follows a similar pattern to that seen in the mouse. Tcf4 is up-regulated during developmental myelination, decreases overtime, and is absent by $7 \mathrm{yr}$ of age. As in the mouse studies, Tcf4 expression reappears in human adult tissue from MS patients, and is specifically expressed in OPCs in active plaques. This expression pattern is suggestive of a poten- tial role for Tcf4-mediated Wnt signaling in the remyelination process. Consistent with this idea, Fancy et al. (2009) demonstrate that DA- $\beta$-catenin signaling is sufficient to impede the remyelination process in mice. As compared with control mice, lesioned tissue from the transgenic mice displayed inhibited recovery and severe hypomyelination, similar to the deficits seen during development. Similar results were obtained in mice lacking a copy of the $\beta$-catenin antagonist APC. Together, these findings suggest that constitutive expression of $\beta$-catenin in OPCs correlates with a significant impairment of the remyelination process. Interestingly, the number of immature OPCs found in demyelinated lesions was unaffected by changes in $\beta$-catenin signaling, suggesting that deficits in remyelination were specifically linked to an inhibition of oligodendrocyte differentiation, and not to defects in progenitor cell migration. The thoroughness and innovative approaches employed by Fancy et al. (2009) provide ample support for the hypothesis that dysregulated Wnt signaling may contribute to the ultimate failure of remyelination, both in mice and in humans.

An important follow-up to the work performed by Fancy et al. (2009) will include further characterization of the factors regulating Tcf4 expression. Wnt-independent pathways have been implicated in the inhibition of Tcf4 expression in OPCs (He et al. 2007). However, the factors promoting Tcf4 expression have not been well characterized (Fig. 1A). Understanding how Tcf4 is up-regulated following demyelination could add greatly to our understanding of the effects of the demyelinated environment on the repair potential of adult OPCs. Are extrinsically expressed Wnt ligands responsible for the post-lesion induction of Tcf4? The answer to this question is intriguing because Wnt ligands represent a secreted source of extrinsic regulation. Previous studies have identified multiple environmental inhibitors of remyelination, including the Notch pathway (Wang et al. 1998; Genoud et al. 2002), PSA-NCAM (Charles et al. 2002), hyaluronan (Back et al. 2005), and LINGO-1 (Mi et al. 2005). With the exception of hyaluronan, these inhibitory cues represent axon-derived membrane-bound factors, implying that cell-cell contact is required to prevent differentiation. In contrast, Wnt ligands represent secreted molecules, which arguably could present an even more extensive capacity for inhibiting differentiation. Fancy et al. (2009) identify changes in the expression of multiple Wnt ligands following the induction of demyelination. Identifying the cells responsible for secreting these ligands could broaden our understanding of the demyelinated environment, in addition to aiding the development of potential therapeutics.

Of course, it cannot be overlooked that the limited capacity for repair exhibited by adult OPCs may result from intrinsic modifications. Recent work suggests that the reduced capacity for regeneration may be due in part to age-related epigenetic changes (Shen et al. 2008). This study suggests that adult OPCs exhibit a decreased efficiency for recruitment of histone deacetylases (HDACs) that ultimately results in permanent inhibition of myelin 
gene expression (He et al. 2007; Shen et al. 2008). Together with the findings of Fancy et al. (2009), these results illustrate the multilayered factors that must be considered in attempts to promote remyelination. While a number of environmental factors have been identified as potent inhibitors of remyelination, intrinsic changes to the OPCs must also be taken into account. Fancy et al. (2009) provide the first in vivo evidence that functional dysregulation of a pathway within OPCs can affect the kinetics of oligodendrocyte differentiation.

\section{The impact of epigenetic regulation}

The findings described here illustrate our burgeoning understanding of the importance of epigenetic changes in both the myelination and remyelination process. As mentioned previously, recent work examining the effect of HDACs on remyelination illustrates the role of chromatin and histone remodeling in development and repair (He et al. 2007; Shen et al. 2008). It is important to remember that these types of chromatin modifications represent only one aspect of epigenetic regulation, which can be defined as changes in gene activity that are not due to modifications of the actual DNA sequence. Indeed, the results from Fancy et al. (2009) illustrate the fundamental importance of examining epigenetic changes on an even broader scale. When considering the results from this

A.

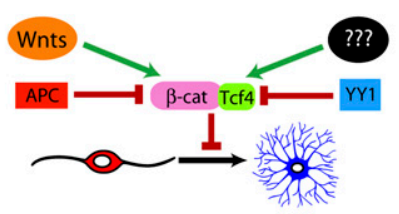

B.

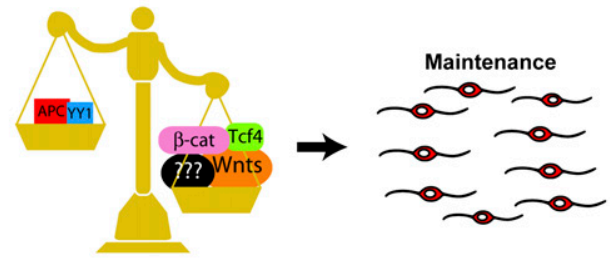

C.

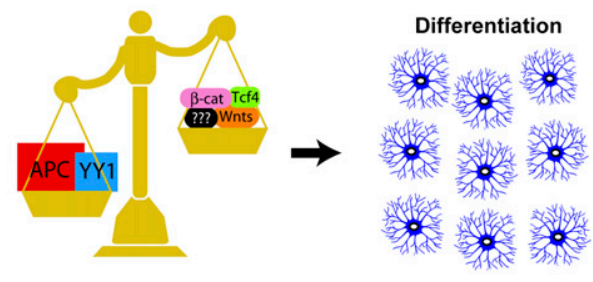

D.

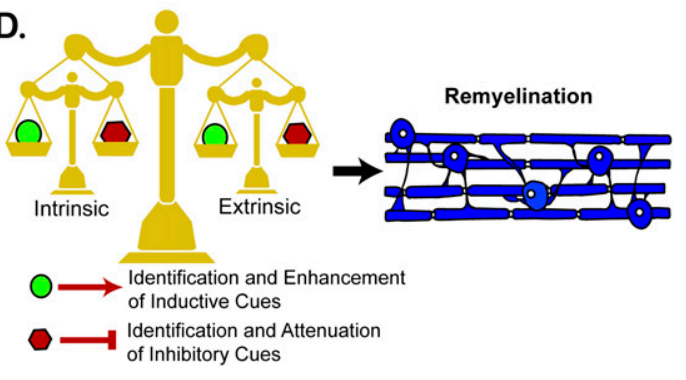

study, one cannot help but be intrigued by the expression pattern of Tcf4. Although expression is measured at various points during development, Fancy et al. (2009) never see $>25 \%$ of the oligodendrocyte lineage expressing Tcf4. As Fancy et al. (2009) succinctly point out, this expression pattern could mean two things. It could point to the existence of a specific subpopulation of OPCs that express Tcf4, or it could mean that Tcf4 expression cycles throughout the entire OPC population and that all cells express Tcf4 at one point or another during development. This latter conclusion is supported by the findings of the study by Fancy et al. (2009). If only a portion of the OPCs were affected by Tcf4/ $\beta$-catenin signaling, one would not expect to see such dramatic delays in differentiation and such a strong hypomyelination phenotype. Indeed, these results suggest that the majority of OPCs are affected by the dysregulation of $\beta$-catenin, supporting a model in which Tcf4 expression occurs in the entire OPC population.

Figure 1. $(A)$ The nucleoprotein transcriptional complex formed between $\beta$-catenin and Tcf4 regulates the active transcription of downstream Wnt target genes. Canonical Wnt signaling mediated through the formation of this complex is sufficient to inhibit the differentiation of OPCs into mature myelinating oligodendrocytes. The formation of this complex is subject to regulation by other factors. APC is an antagonist of $\beta$-catenin expression. Wnt ligands bind to receptors on the surface of OPCs that promote the stabilization of $\beta$-catenin. Yin Yang 1 (YY1) is a transcription factor that inhibits the expression of Tcf4 (He et al. 2007). Although not yet characterized, it will be important to identify the factors that are responsible for promoting Tcf4 expression in OPCs. (B) The expression of factors mediating canonical Wnt signaling determines the fate of oligodendrocytes, both during development and after demyelination. The formation of the $\beta$-catenin/Tcf4 complex inhibits oligodendrocyte differentiation. Binding of Wnt ligands to OPCs promotes the stabilization of the $\beta$-catenin/Tcf4 complex, maintaining the OPCs in an undifferentiated state. Uncharacterized factors that promote Tcf 4 expression in OPCs could also push the cells to remain as nonmyelinating precursors. $(C)$ In contrast, factors that inhibit $\beta$-catenin stabilization and/or Tcf4 expression push the OPCs toward a differentiated fate. APC promotes the degradation of $\beta$-catenin, while YY1 blocks Tcf4 expression. The expression of either of these factors is sufficient to inhibit stabilization of the $\beta$-catenin /Tcf4 complex, tipping the cell fate balance in favor of oligodendrocyte differentiation. $(D)$ The identification of canonical Wnt signaling as a novel regulator of oligodendrocyte cell fate adds to the growing list of cues that must be carefully balanced to promote remyelination. One set of these cues is derived from extrinsic sources, such as inhibitory factors expressed by axons and astrocytes following demyelination. A second level of regulation occurs intrinsically and includes epigenetic regulation as well as the expression of transcription factors that both promote and inhibit oligodendrocyte differentiation. Successful remyelination will require attenuating both the intrinsic and extrinsic inhibitory factors that prevent differentiation. In addition, therapeutic strategies will likely require the induction of cues promoting oligodendrocyte differentiation. A carefully balanced combination of these strategies is essential for promoting repair and improved function. 


\section{A case for heterogeneity?}

The expression pattern of Tcf4 not only presents an interesting puzzle, it also speaks to a fundamental question that must be addressed to understand oligodendrocyte development and myelination. Do OPCs represent a heterogeneous population of cells? If Tcf4 is expressed in all OPCs at some point, why do static time points only show positive staining in less than a quarter of the cells? The myelination field continues to wrestle with the question of heterogeneity in the OPC population. Certainly, developmentally distinct origins from various regions of the CNS argue for the possibility of multiple cell types (Richardson et al. 2006). However, distinctions based on function or expression patterns have been much harder to establish. Indeed, cells from different developmental origins can easily replace one another, arguing against the case for functional differences based on birthplace (Kessaris et al. 2006). This brings up an additional question: If the evidence for heterogeneity is lacking, then are environmental factors responsible for the fact that some OPCs differentiate while others remain precursors into adulthood? Again, a functional distinction between these two populations is not well supported, particularly in light of remyelination studies, which demonstrate the potential for differentiation exhibited by adult OPCs. How, then, can these questions be resolved? How can we explain a heterogeneity of cell fate choices in a population that has not been shown to exhibit heterogeneous patterns of gene expression?

Some insight into this question may be gleaned from the results of Fancy et al. (2009). The expression pattern of Tcf4 could be indicative of the importance of epigenetic regulation in dictating cell fate. The conjecture that all OPCs must eventually express Tcf4 argues for the possibility of temporally mediated fluctuations in protein expression that are independent of changes in gene expression. While immunohistochemical results can detect only a proportion of cells expressing Tcf 4 at a given time, is it possible that more quantitative measurements might reveal Tcf4 expression throughout the precursor cell population, albeit at varying levels of expression? This type of epigenetic variability in protein expression levels has been implicated recently as one factor responsible for the adoption of divergent cell fates in a seemingly homogeneous precursor population (Brock et al. 2009). Perhaps this type of epigenetic regulation could help explain the variability in cell fate experienced by OPCs throughout the CNS. While oligodendrocytes represent a seemingly homogeneous population of cells, the temporal onset of differentiation and myelination is remarkably variable in different regions of the brain. The obvious explanation for this asynchrony is that regional differences in the environment are responsible for the variable timing of differentiation. However, an alternative explanation is that epigenetic variability between OPCs could account for differences in timing. Indeed, myelination is a dynamic process, which in humans can continue into the third decade of life. Oscillations in protein levels could support a model in which interactions of gene regulatory networks keep OPCs in a constant flux until a cell fate is ultimately decided. Stochastic variations in this type of intrinsic regulation could help explain the population-wide variability in the ultimate likelihood of if and when a cell will differentiate. In fact, the idea of a threshold level of expression regulating cell fate decisions is not an entirely novel concept in the myelination field. Previous work has demonstrated that the induction of Schwann cell myelination is regulated by the expression level of neuregulin-1 type III on the surface of the axon (Michailov et al. 2004; Taveggia et al. 2005). While this example represents a ligand receptor-mediated interaction, intrinsic epigenetic regulation could potentially function in a similar manner. Furthermore, not only changes in temporal protein expression levels, but also shifts in subcellular localization may influence cell fate decisions. Indeed, previous work from Rowitch and colleagues (Arnett et al. 2004; Kitada and Rowitch 2006) has demonstrated that OPCs can be distinguished from mature oligodendrocytes based on whether the transcription factor Olig1 is localized to the nucleus or to the cytoplasm, respectively. Obviously, much of OPC differentiation is highly temporally and spatially coordinated by extrinsic factors, as demonstrated both in vivo and in vitro. Therefore, the types of epigenetic regulation discussed here would likely represent simply an added layer of complexity in fine-tuning oligodendrocyte development.

In fact, this type of regulation could help explain another important aspect of the work by Fancy et al. (2009). Fancy et al. (2009) convincingly show that DA- $\beta$ catenin signaling results in delayed differentiation and impaired remyelination. However, the deficit in oligodendrocyte numbers is not accompanied by a corresponding increase in OPC numbers, as is often seen in other studies demonstrating inhibited differentiation (He et al. 2007; Stritt et al. 2009). If these cells are blocked in an undifferentiated state, why are precursor numbers unaffected? Perhaps this result can be clarified in the context of fluctuating levels of gene expression. As Fancy et al. (2009) point out, the expression pattern of the oligodendrocyte lineage is characterized by a continuum of changes in expression, rather than genes being abruptly switched on and off in relation to developmental changes. In fact, it is not unreasonable to suggest that the very definition of a differentiated oligodendrocyte is up for debate. If nothing else, it is safe to say that the line between OPC, premyelinating oligodendrocyte, and newly differentiated oligodendrocyte is murky at best. It is likely, then, as suggested by Fancy et al. (2009), that the reduction in differentiated oligodendrocytes may be due to a block in lineage progression when these cells have progressed beyond precursors but are not yet mature enough to express myelin proteins. Understanding the exact nature and timing of oligodendrocyte lineage transitions may be essential to the treatment of demyelinating conditions. As suggested by the dysregulation hypothesis presented previously, multiple steps could go awry in the remyelination process, and a shift in the timing of any one of them could completely block the possibility of 
repair (Franklin 2002). Therefore, it is essential to remember that a multitude of factors must be considered when approaching the goal of remyelination. However, the complexity should not be cause for discouragement but, on the contrary, should provide cautious optimism.

\section{Where do we go from here?}

Current efforts to promote remyelination highlight the urgent need to become more intimately acquainted with adult OPCs, as they currently represent one of the most promising avenues of effective repair. The myelin field is poised to make significant advances in developing novel therapeutic approaches. We know that adult OPCs are capable of remyelinating, at least to some extent, and we know that these cells are present in demyelinating lesions. Yet the essential issue remains: How do we promote the ongoing differentiation of these cells? The current study by Fancy et al. (2009) provides multiple valuable contributions to addressing this question. First of all, the work by Fancy et al. (2009) validates the importance of Wnt signaling in modulating myelination, both during development and after injury (Fig. 1A-C). This is significant for two reasons. The first is because it shows that pathways involved in developmental myelination may be important in the remyelination process. This supports the idea that attempts to understand myelin from a developmental perspective could contribute to the identification of therapeutic targets for remyelination. The study by Fancy et al. (2009) also shows that pathways involved in myelination and remyelination in the mouse are conserved in humans. These findings support the idea that animal work can truly prove valuable to the treatment of human demyelinating conditions. Finally, the study by Fancy et al. (2009) offers new insight into added layers of complexity that may regulate the myelination process. Our efforts to develop therapeutic strategies can only benefit from a broader understanding of the complex factors involved in the regulation of remyelination (Fig. 1D). The more we understand the players involved, the quicker we can expand our efforts to promote the repair process. The work by Fancy et al. (2009) reminds us that remyelination may depend not only on having the right factors, but on having them in the right place and, of course, at the right time.

\section{Acknowledgments}

We are grateful for the support by the National Multiple Sclerosis Society Career Transition Award to J.R.C. (TA 3008A2/T), the NIH/NINDS to J.R.C. (NS062796-01), and the NIH CBM Predoctoral Training Grant to S.S.R.

\section{References}

Arnett HA, Fancy SP, Alberta JA, Zhao C, Plant SR, Kaing S, Raine CS, Rowitch DH, Franklin RJ, Stiles CD. 2004. bHLH transcription factor Olig1 is required to repair demyelinated lesions in the CNS. Science 306: 2111-2115.

Back S, Tuohy T, Chen H, Wallingford N, Craig A, Struve J, Luo N, Banine F, Liu Y, Chang A, et al. 2005. Hyaluronan accumulates in demyelinated lesions and inhibits oligodendrocyte progenitor maturation. Nat Med 11: 966-972.

Brock A, Chang H, Huang S. 2009. Non-genetic heterogeneityA mutation-independent driving force for the somatic evolution of tumours. Nat Rev Genet 10: 336-342.

Chari D, Blakemore W. 2002. Efficient recolonisation of progenitor-depleted areas of the CNS by adult oligodendrocyte progenitor cells. Glia 37: 307-313.

Charles P, Reynolds R, Seilhean D, Rougon G, Aigrot MS, Niezgoda A, Zalc B, Lubetzki C. 2002. Re-expression of PSA-NCAM by demyelinated axons: An inhibitor of remyelination in multiple sclerosis? Brain 125: 1972-1979.

Dawson MR, Polito A, Levine JM, Reynolds R. 2003. NG2expressing glial progenitor cells: An abundant and widespread population of cycling cells in the adult rat CNS. Mol Cell Neurosci 24: 476-488.

Fancy SPJ, Baranzini SE, Zhao C, Yuk D-I, Irvine K-A, Kaing S, Sanai N, Franklin RJM, Rowitch DH. 2009. Dysregulation of the Wnt pathway inhibits timely myelination and remyelination in the mammalian CNS. Genes \& Dev (this issue). doi: $10.1101 / \mathrm{gad} .1806309$.

Franklin RJ. 2002. Why does remyelination fail in multiple sclerosis? Nat Rev Neurosci 3: 705-714.

Genoud S, Lappe-Siefke C, Goebbels S, Radtke F, Aguet M, Scherer SS, Suter U, Nave KA, Mantei N. 2002. Notch1 control of oligodendrocyte differentiation in the spinal cord. J Cell Biol 158: 709-718.

Gensert JM, Goldman JE. 1997. Endogenous progenitors remyelinate demyelinated axons in the adult CNS. Neuron 19: 197-203.

He Y, Dupree J, Wang J, Sandoval J, Li J, Liu H, Shi Y, Nave KA, Casaccia-Bonnefil P. 2007. The transcription factor Yin Yang 1 is essential for oligodendrocyte progenitor differentiation. Neuron 55: 217-230.

Horner PJ, Power AE, Kempermann G, Kuhn HG, Palmer TD, Winkler J, Thal LJ, Gage FH. 2000. Proliferation and differentiation of progenitor cells throughout the intact adult rat spinal cord. J Neurosci 20: 2218-2228.

Kessaris N, Fogarty M, Iannarelli P, Grist M, Wegner M, Richardson WD. 2006. Competing waves of oligodendrocytes in the forebrain and postnatal elimination of an embryonic lineage. Nat Neurosci 9: 173-179.

Kitada M, Rowitch D. 2006. Transcription factor co-expression patterns indicate heterogeneity of oligodendroglial subpopulations in adult spinal cord. Glia 54: 35-46.

Kotter MR, Li WW, Zhao C, Franklin R. 2006. Myelin impairs CNS remyelination by inhibiting oligodendrocyte precursor cell differentiation. I Neurosci 26: 328-332.

Levine JM, Reynolds R, Fawcett JW. 2001. The oligodendrocyte precursor cell in health and disease. Trends Neurosci 24: 3947.

Levison SW, Young GM, Goldman JE. 1999. Cycling cells in the adult rat neocortex preferentially generate oligodendroglia. J Neurosci Res 57: 435-446.

Mi S, Miller RH, Lee X, Scott ML, Shulag-Morskaya S, Shao Z, Chang J, Thill G, Levesque M, Zhang M, et al. 2005. LINGO-1 negatively regulates myelination by oligodendrocytes. Nat Neurosci 8: 745-751.

Mi S, Hu B, Hahm K, Luo Y, Kam Hui E, Yuan Q, Wong W, Wang L, Su H, Chu T, et al. 2007. LINGO-1 antagonist promotes spinal cord remyelination and axonal integrity in MOGinduced experimental autoimmune encephalomyelitis. Nat Med 13: 1228-1233.

Michailov GV, Sereda MW, Brinkmann BG, Fischer TM, Haug B, Birchmeier C, Role L, Lai C, Schwab MH, Nave KA. 2004. 
Axonal neuregulin-1 regulates myelin sheath thickness. Science 304: 700-703.

Nishiyama A, Chang A, Trapp BD. 1999. NG2+ glial cells: A novel glial cell population in the adult brain. I Neuropathol Exp Neurol 58: 1113-1124.

Richardson WD, Kessaris N, Pringle N. 2006. Oligodendrocyte wars. Nat Rev Neurosci 7: 11-18.

Rivers LE, Young KM, Rizzi M, Jamen F, Psachoulia K, Wade A, Kessaris N, Richardson WD. 2008. PDGFRA/NG2 glia generate myelinating oligodendrocytes and piriform projection neurons in adult mice. Nat Neurosci 11: 1392-1401.

Shen S, Liu A, Li J, Wolubah C, Casaccia-Bonnefil P. 2008. Epigenetic memory loss in aging oligodendrocytes in the corpus callosum. Neurobiol Aging 29: 452-463.

Shimizu T, Kagawa T, Wada T, Muroyama Y, Takada S, Ikenaka K. 2005. Wnt signaling controls the timing of oligodendrocyte development in the spinal cord. Dev Biol 282: 397-410.

Stritt C, Stern S, Harting K, Manke T, Sinske D, Schwarz H, Vingron M, Nordheim A, Knoll B. 2009. Paracrine control of oligodendrocyte differentiation by SRF-directed neuronal gene expression. Nat Neurosci 12: 418-427.

Taveggia C, Zanazzi G, Petrylak A, Yano H, Rosenbluth J, Einheber S, Xu X, Esper RM, Loeb JA, Shrager P, et al. 2005. Neuregulin-1 type III determines the ensheathment fate of axons. Neuron 47: 681-694.

van de Wetering M, Sancho E, Verweij C, de Lau W, Oving I, Hurlstone A, van der Horn K, Batlle E, Coudreuse D, Haramis AP, et al. 2002. The $\beta$-catenin/TCF-4 complex imposes a crypt progenitor phenotype on colorectal cancer cells. Cell 111: 241-250.

Vargas ME, Barres BA. 2007. Why is Wallerian degeneration in the CNS so slow? Annu Rev Neurosci 30: 153-179.

Wang S, Sdrulla AD, diSibio G, Bush G, Nofziger D, Hicks C, Weinmaster G, Barres BA. 1998. Notch receptor activation inhibits oligodendrocyte differentiation. Neuron 21: 63-75.

Watanabe M, Toyama Y, Nishiyama A. 2002. Differentiation of proliferated NG2-positive glial progenitor cells in a remyelinating lesion. J Neurosci Res 69: 826-836.

Windrem M, Nunes M, Rashbaum W, Schwartz T, Goodman R, Mckhann G, Roy N, Goldman S. 2004. Fetal and adult human oligodendrocyte progenitor cell isolates myelinate the congenitally dysmyelinated brain. Nat Med 10: 93-97. 


\section{Erratum}

Genes \& Development 23: 1487-1493 (2009)

Modulating myelination: knowing when to say Wnt

Sheila S. Rosenberg and Jonah R. Chan

In the above-mentioned paper, an incorrect reference was listed in the Reference section for the citation of Shen et al. 2008. The cited reference should have been as follows:

Shen S, Sandoval J, Swiss VA, Li J, Dupree J, Franklin RJ, Casaccia-Bonnefil P. 2008. Age-dependent epigenetic control of differentiation inhibitors is critical for remyelination efficiency. Nat Neurosci 11: 1024-1034.

We apologize for the error. 


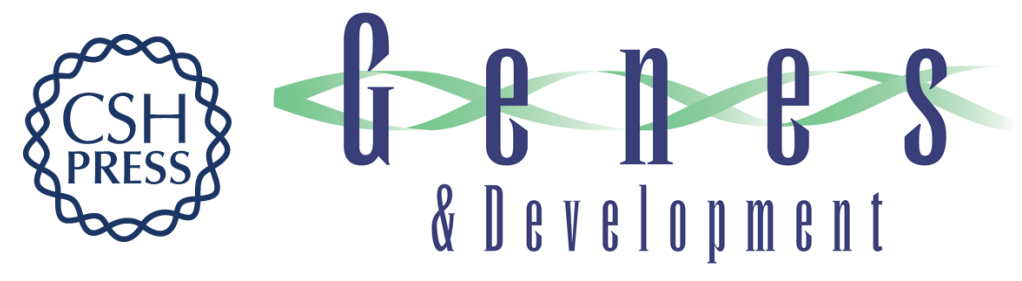

\section{Modulating myelination: knowing when to say Wnt}

Sheila S. Rosenberg and Jonah R. Chan

Genes Dev. 2009, 23:

Access the most recent version at doi:10.1101/gad.1824009

\section{Related Content Dysregulation of the Wnt pathway inhibits timely myelination and remyelination in the mammalian CNS \\ Stephen P.J. Fancy, Sergio E. Baranzini, Chao Zhao, et al. \\ Genes Dev. July, 2009 23: 1571-1585 Erratum: Modulating myelination: knowing when to say Wnt \\ Sheila S. Rosenberg and Jonah R. Chan \\ Genes Dev. August , 2009 23: 1980 \\ References This article cites 32 articles, 5 of which can be accessed free at: \\ http://genesdev.cshlp.org/content/23/13/1487.full.html\#ref-list-1 \\ Articles cited in: \\ http://genesdev.cshlp.org/content/23/13/1487.full.html\#related-urls \\ License Email Alerting
Service $\begin{aligned} & \text { Receive free email alerts when new articles cite this article - sign up in the box at the top } \\ & \text { right corner of the article or click here. }\end{aligned}$}

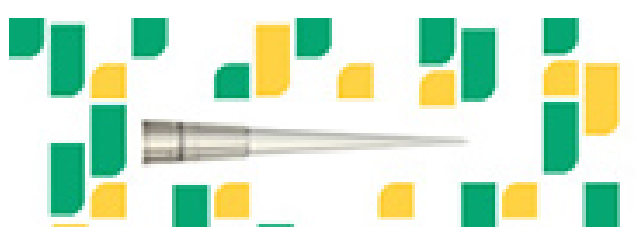

Focused on your science. 